\title{
Mujeres y Carreras de Ingeniería en la Universidad Autónoma de Nuevo León, en México: una Mirada desde las Vivencias de las Estudiantes
}

\author{
Nivia T. Álvarez-Aguilar, Valeria P González-Duéñez y Jaime A Castillo-Elizondo \\ Universidad Autónoma de Nuevo León. Facultad de Ingeniería Mecánica y Eléctrica, México \\ (e-mail: nivial12@yahoo.es; valeria.gonzalezdn@uanl.edu.mx; Jaime.castilloe@uanl.mx)
}

Recibido Dic. 26, 2018; Aceptado Mar. 7, 2019; Versión final Mar. 27, 2019, Publicado Ago. 2019

\begin{abstract}
Resumen
Este artículo tiene como objetivo analizar los factores sociales y personales que inciden en la elección, tiempo de estudios y proyecciones laborales futuras de mujeres en diferentes carreras de ingeniería. Se estudian sus percepciones, en una facultad donde el porcentaje de mujeres es muy bajo con respecto a los hombres. La metodología se corresponde con una investigación no experimental, aplicando un cuestionario con preguntas cerradas de opción múltiple, y tipo Likert dirigido a estudiantes mujeres de nueve carreras de ingenierías de la Universidad Autónoma de Nuevo León, en México. El análisis de los resultados de la encuesta aplicada muestra similitudes con investigaciones recientes en cuanto a los factores que influyen en la selección de estas carreras, así como en la percepción de cierto grado de rechazo, y de diferencias con respecto a los hombres. También afloró la inseguridad acerca del futuro laboral de las participantes en el estudio.
\end{abstract}

Palabras clave: carreras de ingeniería; mujeres estudiantes; género; mujeres en ingeniería; educación superior

\section{Women and Engineering Careers at the University Autónoma de Nuevo León, in Mexico: A View from the Experiences of Woman Students}

\begin{abstract}
The objective of this article is to analyze the social and personal factors that influence the choice, study time and future labor projections of women in different engineering careers. Their perception, in a faculty where the percentage of women is very low compare to men is analyzed. The methodology corresponds to a nonexperimental research, applying a survey with close multiple-choice questions of Linkert type to female students of nine engineering careers from the University Autónoma of Nuevo León, in Mexico. The analysis of the results of the applied survey shows similarities with recent research regarding the factors that influence the selection of these careers, as well as the perception of some degree of rejection and differences with men. The uncertainty about the future employment of the participants in the study was also mentioned.
\end{abstract}

Keywords: engineering careers; female students; gender; women in engineering; higher education 


\section{INTRODUCCIÓN}

En los últimos tiempos en México se ha observado un incremento significativo de la cantidad de mujeres que ingresan a la Educación Superior, no obstante como indican (Oliveros et al., 2016) "solo corresponde al sexo femenino alrededor de $30 \%$ de la población total de estudiantes que cursan algún programa relacionado con ingeniería". (pp. 89). El porcentaje de estudiantes de mujeres y hombres en el año 2016 según la Red de Indicadores de Ciencia y Tecnología (2017) fue de 49,86\% y 50,14\% respectivamente de un total de 4.244.401 egresados, cifras que dan cuenta de una insignificante diferencia de género. Diferentes investigaciones muestran resultados similares como es el caso de la realizada por (Wallace et al., 2012) donde se observa un gran desbalance en cuanto a género en estas carreras. No obstante, el hecho de que se haya incrementado la cantidad de mujeres en la Educación Superior como indicador cuantitativo no debe conllevar a pensar que se ha logrado una equidad de género. Según López y Lizardi (2016) la cantidad de mujeres ha crecido con mayor rapidez que la masculina debido a: factores como la movilidad social, el aumento de las posibilidades económicas y las políticas de equidad con este objetivo, entre los factores que a nuestra consideración se pudieran añadir está el vertiginoso desarrollo de la ciencia y la tecnología.

El concepto de género ha sido ampliamente estudiado por las ciencias sociales. En este sentido, Melero (2010) expresa que el concepto de género y su asociación a la igualdad entre mujeres y hombres, en la actualidad es objeto de debate en diversos medios de la sociedad. A su vez, diferentes estudios han abordado el enfoque de género en la educación en ingeniería. En este sentido, Ihsen (2005) hace énfasis en que este enfoque significa aceptar motivaciones y experiencias de los estudiantes, especialmente de las mujeres. Como expresa Espejo (2015) en el ámbito universitario existen diversas formas de inequidad de género ya sea directa o indirectamente, situación que trae aparejada rechazos, indiferencias, discriminación y abuso con sus subsiguientes consecuencias negativas. Es interesante lo que expresan (Ballarin y Aguado, 2018) al señalar que las políticas de igualdad empiezan a surtir efecto, pero al mismo tiempo han crecido las denuncias de desigualdad laboral, acosos en el trabajo y de manifestaciones machistas a nivel mundial. Tanto es así que en el análisis de una base de datos internacional realizado por Stoet y Geary (2018), en casi todos los países las estudiantes mujeres mostraban más capacidad para el estudio de las carreras STEM. Cada día aumenta el interés por las carreras universitarias en áreas de Ciencia, tecnología, ingenierías y matemáticas (STEM) debido a que dichas áreas están marcando las tendencias en el desarrollo científico tecnológico de la actualidad. Avendaño y Magaña (2018) muestran algunas tendencias a través de un estado de conocimientos sobre la elección de estas carreras. Sin embargo, la equidad de género constituye un gran reto para el logro de la inclusión en el sistema educativo mexicano.

En los últimos años han proliferado las investigaciones que abordan la situación de la mujer en las carreras tradicionalmente consideradas como masculinas. Se ha incrementado paulatinamente la incorporación a dichas carreras, aunque se reconocen los avances, aún quedan muchos vacíos que se relacionan con aspectos personales, familiares y sociales. De igual forma la poca presencia de la mujer en carreras de ingeniería es una manifestación de los prejuicios sociales en el momento de la elección de la carrera. A pesar del acelerado desarrollo de la Ciencia y la Tecnología y las oportunidades de desarrollo que ofrecen las carreras que guardan relación con él, todavía no se ha logrado comprender que no son carreras "para hombres" solamente. También cobra importancia la participación de la mujer en las citadas carreras, diferentes estudios muestran que en la actualidad persisten los obstáculos que enfrentan las mujeres que estudian carreras de ingeniería. (Salinas y Romaní, 2016), describen las tensiones percibidas por las mujeres en su tiempo de estudios así como sus temores para su inserción en el mundo laboral una vez graduadas. Las mujeres constituyen minoría en carreras tecnológicas y de ingeniería porque se conjugan múltiples factores sociales que condicionan su elección. En este caso, diferentes autores como lto y McPherson (2018) han encontrado una influencia significativa de los factores sociales en las percepciones de las mujeres hacia carreras de STEM.

Entre los factores sociales persiste el machismo como uno de los que más influye, (Lorenzo et al., 2016) señalan que el grupo de hombres que participó en el estudio realiza aseveraciones fundamentadas en este tipo de estereotipo y destacan una serie de cualidades que según ellos son propias de los hombres. Sin embargo, este tema ha sido objeto de estudio desde algunas décadas, incluso relacionando la participación de la mujer con inclusión en las políticas de estado (Kudva, 2003). Por otra parte, (Ortman, 2015) destaca que los estereotipos y desigualdades presentes en toda la trayectoria escolar de las alumnas desde que comienzan en la escuela van conformando un ambiente desfavorable que influye negativamente en el ingreso y permanencia en las carreras de ciencia. En este mismo sentido, (Cheryan et al., 2015) señalan que en la cultura americana persisten estereotipos que influyen la selección por parte de las mujeres de carreras de computación e ingeniería ya que se considera que el tipo de trabajo y las características de dichas carreras son más afines a los hombres. 
Otros autores como Álvarez et al., 2014, achacan la insuficiente motivación de las alumnas por carreras de ingeniería entre otros factores porque consideran que el tener buenas calificaciones no son suficientes para elegir dichas carreras, además cuentan con escasos modelos femeninos en el ámbito científico tecnológico. En investigaciones anteriores (Bell et al., 2003) se han corroborado que los estereotipos de género influyen en los resultados obtenidos particularmente en matemáticas para ingeniería, lo que no significa que la capacidad de las mujeres para estas materias es más baja con respecto a los hombres. Todo este entramado de factores unido a un limitado trabajo de orientación vocacional hacia estas carreras confluye en la elección de las mismas.

Múltiples investigaciones han explorado diferentes factores que han influido y motivado a las estudiantes de ingeniería en la elección de la carrera en general y en particular de las ingenierías. Así por ejemplo, los hallazgos de (Kolmos et al., 2013) muestran resultados interesantes en cuanto a las diferencias en los factores motivacionales de hombres y mujeres en la elección de dichas carreras. La contribución del presente estudio está dada fundamentalmente en precisar los principales factores sociales y personales en un contexto donde se observa que 9 carreras de ingeniería que cuentan con un alto número de estudiantes, el porcentaje de mujeres es muy bajo.

\section{METODOLOGÍA}

El presente estudio se basa en una investigación no experimental ya que de acuerdo a sus objetivos permite encontrar los resultados que puedan aportar conocimientos al problema objeto de estudio. Se utilizó un diseño metodológico descriptivo de corte transversal. Se analiza una realidad concreta cuya amplitud está dada por abarcar 9 carreras de ingenierías. Aunque el estudio se centra en mujeres estudiantes de dichas carreras, los resultados revelan la interacción de ellas con otros sujetos como docentes, directivos y estudiantes hombres, así como con procesos propios de la formación en ingeniería. Se busca llegar a conclusiones a través de las percepciones de las estudiantes mujeres sobre diferentes aspectos de su carrera. Este es el camino que describen Denzin y Lincoln (2012) cuando plantean: "el investigador recaba el material empírico relacionado con el problema y luego produce análisis y escritura sobre ese material".

Para recabar los datos se aplicó un cuestionario con preguntas cerradas de opción múltiple, y tipo Likert dirigido a estudiantes mujeres de 9 carreras de ingenierías de una universidad del Norte de México con el propósito de determinar cómo se perciben las mujeres en carreras de ingeniería en la realidad educativa donde transcurre el proceso formativo. También se utilizaron preguntas abiertas para una mayor libertad al opinar que posteriormente se agruparon en categorías para su cuantificación. Las categorías fueron determinadas tanto por la revisión de las fuentes como por las cuestiones vivenciadas por las estudiantes con mayor frecuencia. La categorización se realizó a través del análisis de las opiniones de las estudiantes que se incluyeron en las tres categorías previamente determinadas que se enuncian a continuación. Todas las preguntas se agruparon en tres categorías para una mejor organización y comprensión de los resultados: 1) Factores que influyeron en la elección de la carrera, entre las preguntas utilizadas en esta categoría estaban: "De los factores mencionados, marca cuáles fueron los de mayor influencia en la elección de su carrera"(opción múltiple), "Menciona las causas por las cuales las mujeres no eligen una carrera de ingeniería" (pregunta abierta), “¿ Volverías a elegir una carrera de ingeniería?" (Pregunta cerrada); 2) Percepciones acerca de su situación como estudiantes se incluyeron: "¿Has percibido diferencias por ser mujer?" (Pregunta cerrada), ¿“Cómo valoras tu relación con los compañeros de clase?" (Pregunta cerrada), “¿Cómo catalogas tu liderazgo en el grupo?" (Pregunta cerrada), y 3) Sus proyecciones de futuro, "Según tu criterio. ¿Existen dificultades para la obtención de puestos una vez graduadas?" (Tipo Likert).

El estudio abarcó 9 carreras de ingeniería de una facultad que tiene 11 carreras. A inicios del 2018 según datos del Sistema integral para la Administración de Servicios Educativos (SIASE), contaba con 18,221 estudiantes, de ellos 14,990 son hombres y 3231 son mujeres que representan el $17,73 \%$ del total. Significa que es muy bajo el \% de mujeres. La población estuvo compuesta de estudiantes que se encontraban cursando del segundo semestre en adelante por considerarse que ya en ese momento podían aportar elementos más fundamentados que las mujeres que se encuentran en los primeros semestres. En la Tabla 1, aparece la población y en la Tabla 2, la muestra utilizada que fue de carácter aleatorio. Es oportuno referir que las estudiantes que participaron en este estudio fueron informadas de su objetivo, estableciéndose un compromiso de confidencialidad por lo que se mantuvo el anonimato, así como el nombre de la universidad.

\section{RESULTADOS Y DISCUSIÓN}

De acuerdo al objetivo propuesto y la metodología utilizada, el análisis de los resultados se ha agrupado en las 3 categorías ya mencionadas. A continuación se exponen los resultados de mayor interés. 
Tabla1: Total de estudiantes mujeres por carrera durante el 2017. Datos obtenidos del (SIASE).

\begin{tabular}{|l|c|c|}
\hline \multicolumn{1}{|c|}{ Carreras } & $\begin{array}{c}\text { Total, de } \\
\text { mujeres }\end{array}$ & $\begin{array}{c}\text { De } 2^{\circ} \text { en } \\
\text { adelante }\end{array}$ \\
\hline Ingeniero Tecnología de Software & 225 & 202 \\
\hline Ingeniero Administrador de Sistemas & 671 & 614 \\
\hline Ingeniero en Electrónica y Automatización & 77 & 74 \\
\hline Ingeniero en Electrónica y Comunicaciones & 95 & 88 \\
\hline Ingeniero en Manufactura & 103 & 88 \\
\hline Ingeniero en Materiales & 134 & 111 \\
\hline Ingeniero Mecánico Administrador & 1048 & 945 \\
\hline Ingeniero en Mecatrónica & 380 & 322 \\
\hline Ingeniero Mecánico Electricista & 104 & 92 \\
\hline Totales & 2837 & 2536 \\
\hline
\end{tabular}

Tabla 2: Muestra de estudiantes participantes en el estudio

\begin{tabular}{|l|c|}
\hline \multicolumn{1}{|c|}{ Carreras } & Cantidad de estudiantes \\
\hline Ingeniero Administrador de Sistemas (IAS) & 90 \\
\hline Ingeniero Electrónico y Automatización (IEA) & 11 \\
\hline Ingeniero Electrónico y Comunicaciones (IEC) & 27 \\
\hline Ingeniero Mecánico Administrador (IMA) & 55 \\
\hline Ingeniero Mecánico Electricista (IME) & 18 \\
\hline Ingeniero en Manufactura (IMF) & 24 \\
\hline Ingeniero en Materiales (IMT ) & 37 \\
\hline Ingeniero en Mecatrónica (IMTC) & 85 \\
\hline Ingeniero en Tecnología de software (ITS) & 42 \\
\hline Total Carreras: 9 & 389 \\
\hline
\end{tabular}

\section{Categoría 1. Factores que influyeron en la elección de la carrera}

La Figura 1 muestra que el factor más influyente en la elección de la carrera fue la familia. Podría parecer una contradicción si se toman en cuenta los prejuicios sociales existentes pero en realidad esta cantidad es menor a la mitad de estudiantes encuestadas. Es oportuno destacar que este resultado contrasta con la investigación sobre "Mujeres: carreras tecnológicas y problemáticas asociadas" realizada por (Morris et al., 2012) donde destacan que a diferencia de otros estudios, las estudiantes participantes no consideraron el factor familiar entre los que más influyeron en su elección. Les siguen en orden los factores relacionados con el prestigio de la facultad y la universidad. Es importante destacar que al estar enclavada la universidad en una zona industrial, las carreras de ingeniería son bien vistas por las posibilidades de empleo una vez graduadas. Al mismo tiempo, al agrupar los medios de comunicación y las TIC, suman 86 estudiantes que eligieron estos factores que sería un factor bastante influyente con respecto a los demás. En el estudio citado se encontró que el mayor por ciento de las mujeres que eligieron carreras de ingeniería y tecnología estuvieron motivadas por el perfil de egreso y las materias (38\% del total). Estos resultados son similares a los obtenidos en el presente estudio ya que "el prestigio de la carrera" según las encuestadas está muy relacionado con su perfil de egreso.

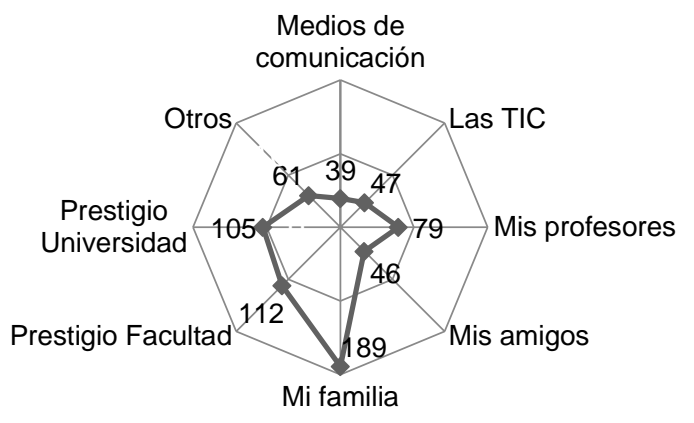

Fig. 1: Nivel de influencia de factores en la elección de la carrera, $n=389$ 
Se realizó una pregunta abierta sobre cuáles eran según su opinión las causas por las que las mujeres no eligen carreras de ingeniería. Las respuestas se agruparon en categorías que aparecen en la Tabla 3. Como se observa en la tabla, el mayor porciento de respuestas $(22.80 \%)$ se concentró en causas como el machismo, la discriminación y el acoso lo que por supuesto tiene una relación muy estrecha con el grupo de causas que tiene que ver con otros aspectos sociales como los estereotipos y prejuicios sociales, el segundo lugar en las frecuencias de respuestas (18.24\%), señalan concretamente entre estas causas: el que la sociedad piensa que es una carrera para hombres, que tienen miedo a ser mal vistas, el temor a qué dirán, entre otras. Muchas estudiantes indican como obstáculos que influyen en la elección de estas carreras, el grado de dificultad de su campo de estudio y las materias como las matemáticas y la física. Esto constituye una preconcepción errónea ya que los resultados académicos demuestran que las estudiantes una vez que ingresan se desenvuelven sin dificultades. Según las respuestas estas se encuentran entre el tercer grupo de causas que más influyen en la selección (15,96 \% de respuestas).

En la revisión de la literatura aparecen otro grupo de causas relacionadas a cuestiones personales, como: miedo, inseguridad, autoestima, pero a fin de cuentas estas no son más que consecuencias de prejuicios y estereotipos sociales que permean la familia, la escuela y todo el ambiente donde se educan las mujeres. El papel que juega la escuela en la elección de la carrera ha sido considerado como fundamental en particular a través de la orientación vocacional (Barragán, 2017). También es significativa la influencia en la selección de las causas que se relacionan con el trabajo futuro, un porciento nada insignificante de las respuestas (14, $98 \%$ ) se corresponde con este tipo de causas. Por último, se enuncian otras causas muy diversas como: falta de apoyo para estudiar, negación de los padres, porque no es su vocación, por no batallar con las materias, porque temen ensuciarse las manos y otras.

Estos resultados tienen coincidencias con estudios realizados en otros contextos con mujeres estudiantes de secundaria (Álvarez et al., 2014). En este caso, se encontró que también son muy notorios estereotipos de género, las participantes en este estudio sentían que su medio considera que la ingeniería no es un trabajo propio para mujeres, en correspondencia con esto, solamente el $19,8 \%$ consideró que si es propicio para ellas. Por otra parte, la mayoría de las estudiantes encuestadas (Figura 2), expresaron que, si tuvieran que elegir de nuevo la carrera, elegirían la misma, este hecho es un indicador de que su decisión fue bien razonada o también pudiera ser que una vez que conocieron mejor la carrera se sintieron más motivadas.

Tabla 3: Causas por las que las mujeres no eligen carreras de ingeniería.

\begin{tabular}{|l|c|c|}
\hline \multicolumn{1}{|c|}{ Causas } & \# de Resp. & Frecuencia \\
\hline Machismo, discriminación y acoso & 140 & $22.80 \%$ \\
\hline Estereotipos y prejuicios sociales & 112 & $18.24 \%$ \\
\hline $\begin{array}{l}\text { Cuestiones personales: inseguridad, miedo, } \\
\text { autoestima }\end{array}$ & 92 & $14.98 \%$ \\
\hline $\begin{array}{l}\text { Grado de dificultad de las carreras por la } \\
\text { manipulación de objetos, materias muy } \\
\text { difíciles }\end{array}$ & 98 & $15.96 \%$ \\
\hline $\begin{array}{l}\text { Inseguridad y diferencias, dificultad para } \\
\text { encontrar trabajo }\end{array}$ & 92 & $14.98 \%$ \\
\hline $\begin{array}{l}\text { Falta de información y de conocimiento } \\
\text { sobre las carreras }\end{array}$ & 40 & $06.51 \%$ \\
\hline Otras causas & 40 & $06.51 \%$ \\
\hline Total de Resp. & 614 & $100.00 \%$ \\
\hline
\end{tabular}

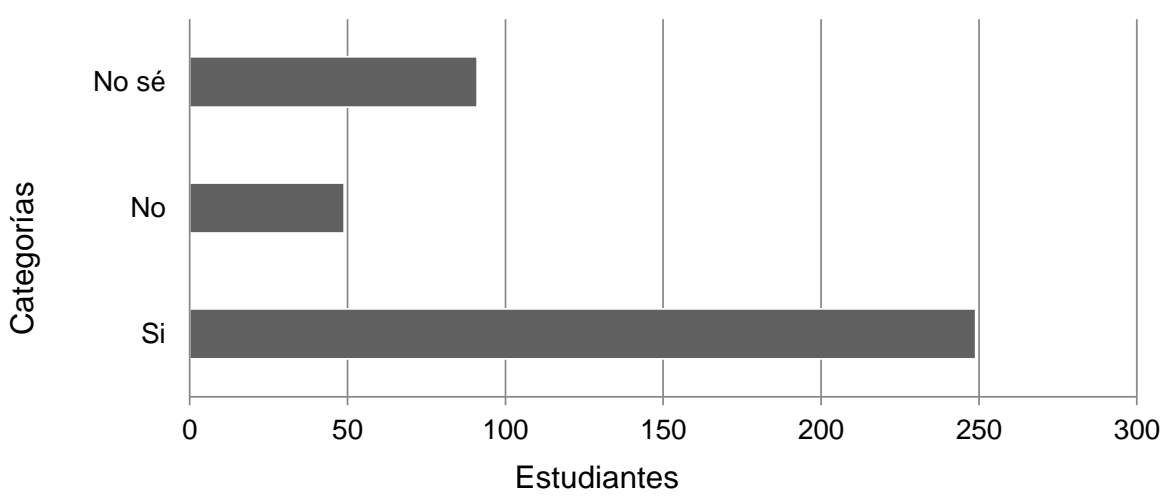

Fig. 2: Opinión sobre una posible segunda elección de la carrera, n=389 


\section{Categoría 2. Percepciones acerca de su situación como estudiantes}

Con respecto a la satisfacción por la carrera una vez que ya están en la facultad, la Figura 3 muestra que la mitad de las estudiantes encuestadas se sienten muy satisfechas, solo el $4 \%$ indica que están insatisfechas. Este resultado coincide con lo que se observa en la Figura 2 sobre el nivel de seguridad que expresaron acerca de su elección.

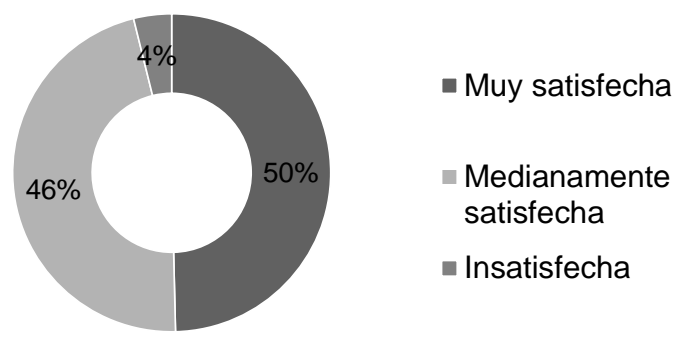

Fig. 3: Grado de satisfacción por la carrera, n=389

En la pregunta que indaga sobre el posible rechazo por ser mujer (Figura 4), aunque el $66 \%$ refiere que "no", no es despreciable la cantidad de estudiantes que señalan que "si" y "en parte". Al sumar estas dos categorías se obtiene dato significativo, un $34 \%$. No es igual "sentir diferencia" que "sentir rechazo" por eso se indagó si las estudiantes habían sentido diferencia por ser mujeres, se obtuvieron resultados similares, pero se muestra más aguda la percepción de diferencia ya que como a parecer en la Figura 5, el $66 \%$ incluye las que expresaron que "sí" y "en parte". Investigaciones han demostrado que en la educación en ingeniería, los trabajos en equipo presentan diferencia de género. De acuerdo con (Beddoes y Panther, 2018) los profesores requieren de herramientas para el logro de la inclusión y la equidad de género en este tipo de trabajo. Se está de acuerdo con este criterio ya que es lógica la relación estrecha entre el trabajo del profesor y el nivel de satisfacción de los estudiantes por su carrera.
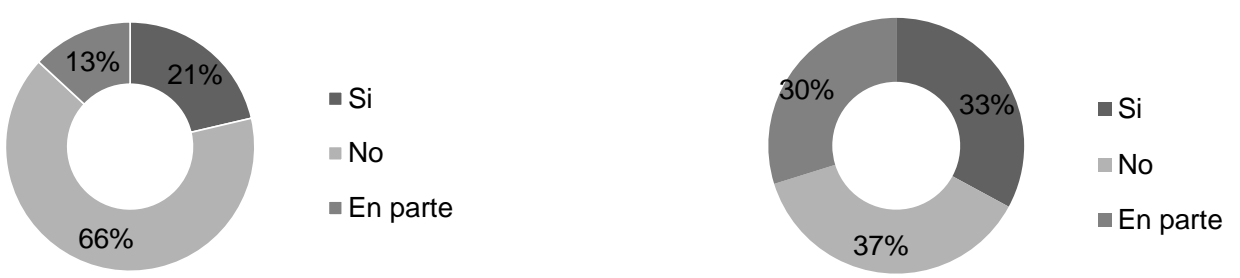

Fig. 4: Sensación de rechazo por ser mujer, $n=389$

Fig. 5: Percepción de diferencia por ser mujer, $n=389$

Uno de los indicadores que puede ser de interés en la determinación de la presencia de diferencias de género es la comunicación con los compañeros de clases (Figura 6). En este caso, más de la mitad de las encuestadas, el $56 \%$ considera que es "buena" y solo el $37 \%$ manifiesta que es "excelente". Este hecho ha sido tomado en cuenta en diferentes investigaciones como es el caso de la realizada por (Walton et al., 2015) quienes realizaron experimentos que incluyeron intervenciones para disminuir el estrés y otras situaciones a las que están expuestas las mujeres que estudian carreras de ingeniería con resultados positivos. De igual manera, Dennehya y Dasgupta (2017) desarrollaron una intervención con mujeres que estudian carreras de ingenierías aplicando la mentoría entre pares del género femenino, los datos obtenidos en este estudio permitieron concluir que dicha intervención fue efectiva en cuanto a la confianza en sí mismas, la motivación y la retención en la carrera.

Diferentes estudios como el realizado por Gutiérrez $(2015$, p.17) sobre el estilo de liderazgo, formas de comunicación y otras cuestiones relacionadas con las diferencias entre hombres y mujeres asienten afirman "que las mujeres se caracterizarían por ejercer un liderazgo con mayor énfasis en la visión de la empresa u organización". En este caso, se observa coincidencia con este criterio ya que las propias estudiantes opinaron que presentan liderazgo. La mayor cantidad de estudiantes (Figura 7) respondió que "si", (36\%) una parte considerable no estaba segura, al responder "no sé" (34 \%), y la minoría respondió que "no" $(30 \%)$. Este es un factor que tiene influencia favorable a las estudiantes en su tiempo de estudios. Relacionado con lo anterior en el trabajo de (Oliveros et al., 2016), al preguntarles a las estudiantes ¿Cuál es tu meta al estudiar ingeniería? El 59 \% indicó que "demostrar que pueden", es interesante que en esta opinión aunque subyacen los estereotipos y mitos que hemos mencionado, en lugar de disminuirlas les hacen sentir la necesidad de demostrar que pueden lograr sus metas. En estudios realizados (Haworth et al., 2010) con infantes y adolescentes se ha comprobado que las estudiantes pueden presentar un mejor rendimiento en los cursos de ciencia a diferencia de los varones. 


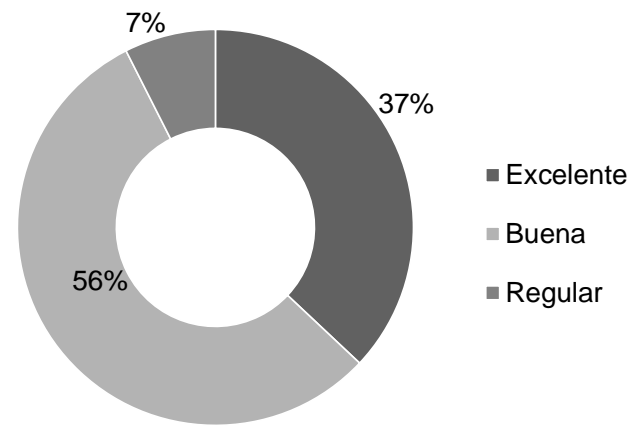

Fig. 6: Relaciones con los compañeros de clases

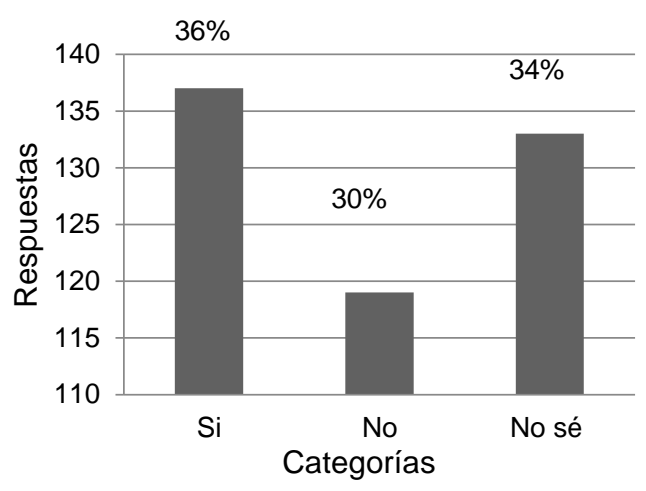

Fig. 7: Autovaloración de liderazgo en el grupo.

\section{Categoría 3. Proyección de futuro}

Fue de interés indagar algunos aspectos relacionados con la proyección de futuro de las estudiantes participantes en el estudio. Al preguntarles si consideraban que su condición de ser mujer influía en el posicionamiento de altos puestos (Figura 8), la mayoría estuvo "totalmente de acuerdo" (35\%), el (23\%) estuvo "de acuerdo" y solamente el (11\%) manifestó estar "en desacuerdo" o "totalmente en desacuerdo". Estos datos se corresponden plenamente con lo expresado por diferentes autores que han estudiado el tema, tal y como fue referido en la introducción de este trabajo. Estos resultados son congruentes con trabajos actuales como el realizado por (Martínez, 2018) quien expresa que "la presencia de la mujer en el ámbito profesional sobre todo en profesiones como la ingeniería que ha sido considerada como de corte masculino". (p.147). Han sido ampliamente reconocidas las diferencias de género relacionadas con el trabajo propio de ingeniería que según Fox (2010) puede mejorarse con diferentes políticas institucionales que favorezcan la igualdad.

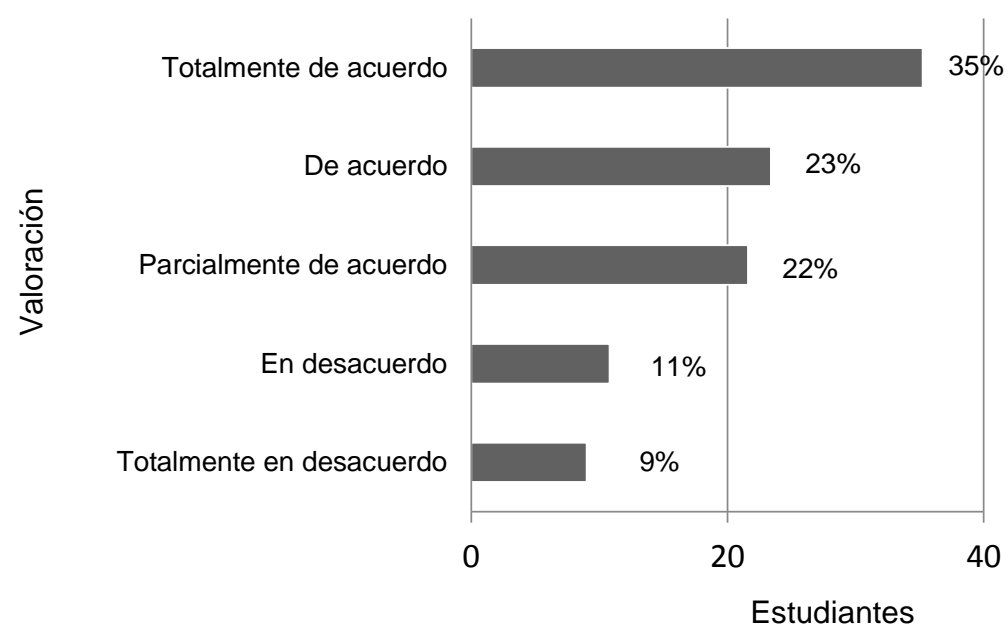

Fig. 8: Dificultades en la obtención de altos puestos por ser mujeres según percepción de estudiantes

Los resultados obtenidos en este estudio tienen implicancia en la necesidad de continuar investigando acerca de los obstáculos a vencer en cuanto a la participación de la mujer en carreras de ingeniería, tema que abarca muchos factores que incluyen a las propias mujeres, quienes deben superar mitos y estereotipos que aún persisten. Resultados de investigación en la Universidad de Utrecht (Dilli y Westerhuis, 2017) afirman que eliminar las brechas de género en las ciencias dando la misma prioridad a las mujeres que a los hombre estimula la actividad innovadora en las empresas. Los principales resultados obtenidos permitieron dar cumplimento al objetivo propuesto al destacarse aquellos factores sociales y personales que tuvieron una determinada implicación en la selección de la carrera, el tiempo de estudio de estudiantes y sus proyecciones futuras. Aunque muchos de los hallazgos coincidieron con los encontrados en estudios anteriores, se considera de interés continuar dando seguimiento de acuerdo a los cambios que van ocurriendo en la sociedad. La mayor aportación de presente trabajo está dada en el valor educativo del análisis realizado con el fin de proyectar acciones en el contexto donde se realizó el estudio. No obstante, vale destacar que se pudiera considerar una limitación la relacionada con la importancia de ampliar este estudio a estudiantes hombres lo que permitiría realizar comparaciones que proporcionarían aportaciones útiles e interesantes al tema investigado. 


\section{CONCLUSIONES}

De acuerdo a los resultados de este estudio, y de su discusión y análisis se elaboran las conclusiones principales siguientes:

1) La literatura sobre el tema abordado permite comprender que están ocurriendo avances en la igualdad de oportunidades para ambos sexos en cuanto a las posibilidades para ingresar en la Educación Superior, incluso en muchos casos la matrícula de mujeres es mayor que la de hombres.

2) Se coincide con la literatura consultada en que la presencia de la mujer en las carreras de Ciencia y Tecnología, en particular en ingenierías, aún es escasa, regularidad observada en este estudio.

3) Resultados obtenidos en investigaciones de otros países muestran que persisten los mitos y estereotipos sociales que influyen en los diferentes contextos donde se desenvuelven las estudiantes mujeres, principalmente en las instituciones educativas, la familia y en la sociedad en general.

4) Es notoria la diferencia entre la percepción de las mujeres antes de elegir la carrera y una vez que han comenzado sus estudios. Comparación que ha sido insuficientemente estudiada.

5) En la investigación realizada se percibe un buen nivel de satisfacción de las estudiantes mujeres por la carrera elegida. Asimismo, la mayoría de las encuestadas aprecia que cuenta con liderazgo entre sus compañeros, a pesar de que distinguen cierto nivel de rechazo por ser mujeres y además tienen la sensación que no es un trato igualitario con respecto a los estudiantes hombres.

\section{REFERENCIAS}

Álvarez, J., A. Arias, F. Serrallé y M. Varela, Elección de Estudios de Ingeniería: Influencia de la Educación Científica y de los Estereotipos de Género en la Autoestima de las Alumnas, Rev. Investigación en Educación, 12 (1), $54-72$ (2014)

Avendaño, K.C. y D.E. Magaña, Elección de Carreras Universitarias en Áreas de Ciencia, Tecnología, Ingeniería y Matemáticas (STEM), Revista Interamericana de Educación de Adultos CREFAL, 40(2), 155-173 (2018)

Ballarín, P. y E. Aguado, Mujeres Rompiendo la División Sexual de los Espacios Laborales, doi: 10.18002/cg.v0i13.5467, Cuestiones de género: de la igualdad y la diferencia,1(13), 1-6 (2018)

Barragán, M., School and Institutional Effects on Secondary Education Transitions in Mexico, doi:10.1016/j.ijer.2017.06.005, International Journal of Educational Research, 85, 68-86, (2017)

Beddoes K. y G. Panther, Gender and Teamwork: An Analysis of Professors' Perspectives and Practices, doi: 10.1080/03043797.2017.1367759, European Journal of Engineering Education, 43(3), 330-343 (2018)

Bell, A., S. Spencer, E. Iserman y C. Logel, Stereotype Threat and Women's Performance in Engineering, doi: 10.1002/j.2168-9830.2003.tb00774.x, Journal of Engineering Education, 92 (4), 307-312 (2003)

Cheryan S., A. Master y A.N. Meltzoff, Cultural Stereotypes as Gatekeepers: Increasing Girls' Interest in Computer Science and Engineering by Diversifying Stereotypes, doi: 10.3389/fpsyg.2015.00049, Frontiers in Psychology, 6(49), 18 (2015)

Dennehya T.C. y N. Dasgupta, Female Peer Mentors Early in College Increase Women's Positive Academic Experiences and Retention in Engineering, doi: 10.1073/pnas.1613117114, Proceedings of the National Academy of Sciences of the United States of America (PNAS), 114 (23), 5964-5969 (2017)

Denzin, N. e Y. Lincoln, Introducción General. La Investigación Cualitativa como Disciplina y como Práctica, En: El Campo de la Investigación Cualitativa. Manual de Investigación Cualitativa, Gedisa, 1, 43-101, Barcelona, España (2012)

Dilli S. y G. Westerhuis, How Institutions and Gender Differences in Education Shape Entrepreneurial Ativity: a CrossNational Perspective, doi: 10.1007/s11187-018-0004-x, Small Business Economics, 51, 371-392 (2018)

Espejo, R., Interacción Simbólica en un Acto de Subjetividad de Género, doi: 10.4067/S0718- 50062015000500006, Formación Universitaria 8(5) 43-58 (2015)

Fox, M. F., Women and Men Faculty in Academic Science and Engineering: Social-organizational Indicators and Implications, American Behavioral Scientist, 53(7), 997-1012 (2010)

Gutiérrez, O., Estudios de Liderazgo de Hombres y Mujeres, doi: 10.26797/rpye.v0i126.62, Revista Política y Estrategia, 1(126), 13-35 (2015)

Haworth, C., P. Dale y R. Plomin, Sex Differences in School Science Performance from Middle Childhood to Early Adolescence, doi: 10.1016/j.jijer.2010.09.003, International Journal of Educational Research, 49, 92-101 (2010)

Insen, S., Special gender studies for engineering, doi: 10.1080/03043790500213144, European Journal of Engineering Education,30 (4) 487-494 (2005)

Ito, T. y E. McPherson, Factors Influencing High School Students Interest in PSTEM, doi: 10.3389/fpsyg.2018.01535, Frontiers in Psychology, 9, (2018) 
Kolmos, A., Mejlgaard, N., Haase, S. y E. Holgaard, Motivational factors, gender and engineering education, doi: 10.1080/03043797.2013.794198, Journal European Journal of Engineering Education, 38(3), 340-358 (2013)

Kudva, N., Engineering Elections: The Experiences of Women in Panchayati Raj in Karnataka, India, International Journal of Politics, Culture and Society, 16, 445-463 (2003)

López, M. y V. Lizardi, La Mujer Mexicana Educada en Valores para su Inserción Laboral sin Discriminación, Opción, 32(13), 815-839 (2016)

Lorenzo, M.I., F.J. Álvarez, M. Álvarez y J.F. Serrallé, La Amenaza del Estereotipo: Elección de Estudios de Ingeniería y Educación Tecnocientífica, Opción, 32(9), 54-76 (2016)

Martínez, K.I., Mujeres en Profesiones Masculinas. El Caso de las Ingenieras Mecánicas Electricistas. ¿Mujeres florero?, doi: 10.20318/femeris.2018.4079, Femeris, 3(1),147- 160 (2018)

Melero, N., Reivindicar la igualdad de mujeres y hombres en la sociedad: una aproximación al concepto de género, doi: http://dx.doi.org/10.20932/barataria.v0i11.152, Revista Castellano-Manchega de Ciencias Sociales, 11, 73-83 (2010)

Montero, M.C., M.S. Porris, L. Amado y M.M. Marinsalta, Foro Mundial de Educación en Ingeniería - WEEF, Mujeres: Carreras Tecnológicas y Problemáticas Asociadas, Buenos Aires, Argentina (2012)

Oliveros, M.A., E. Cabrera, B. Valdez y W. Schorr, La Motivación de las Mujeres por las Carreras de Ingeniería y Tecnología, doi: 10.21933/J.EDSC.2016.09.157, Entreciencias, 4(9), 89-96 (2016)

Ortmann, C., Mujeres, Ciencia y Tecnología en las Universidades: ¿la Excepción a la Regla? Procesos de Construcción Identitaria Profesional en Estudiantes de Ingeniería, Revista del IICE 1(38), 95-107 (2015)

Red de indicadores de Ciencia y Tecnología (RYCIT), Títulos de Grado 2006-2015 (2017)

Salinas, P. y G. Romani, Proyección Laboral de las Estudiantes Mujeres en Carreras Mineras en la Educación Superior Chilena, doi: 10.4067/S0718-50062017000300005, Formación Universitaria, 10(3), 31-48 (2016)

Stoet G. y D. Geary, The Gender-Equality Paradox in Science, Technology, Engineering, and Mathematics Education, doi: 10.1177/0956797617741719, Psychological Science, 29(4), 581-593 (2018)

Wallace, M., I. Lings, N. Sheldon y R. Cameron, Female Engineering Students: Career Attractors, GIEE 2011: Gender and Interdisciplinary Education for Engineers, Sense Publishers, 223-240 (2012)

Walton, G. M., C. Logel, J.M. Peach, S.J. Spencer y M.P. Zanna, Two brief Interventions to Mitigate a "Chilly Climate" Transform Women's Experience, Relationships, and Achievement in Engineering, doi: 10.1037/a0037461, Journal of Educational Psychology, 107(2), 468-485 (2015) 
